\title{
Real-Time Intelligent Control Systems
}

This special issue of Integrated Computer-Aided Engineering is devoted to Integration Technology for Real-Time Intelligent Control Systems. Although specific intelligent control techniques are analyzed and developed in many articles, the aim of this issue is to insist on their integration, this step often being the bottleneck between laboratory prototypes and large scale production systems. Moreover, effective intelligent control systems are essentially heterogeneous, since they have to integrate a variety of techniques ranging from model-based reasoning to fuzzy logic or neural networks.

In this issue, you will find a set of articles that cover the main aspects of this integration task from three different, but closely related, viewpoints: real time integration architectures, integration methodologies and basic intelligent control technologies. Integration architectures cope with the global design of applications, assuming that they are composed by a set of interleaved elements. The architecture specifies the role of each subsystem, as well as the interactions among them. Integration methodologies try to make efficient the interchange of data and services among subsystems. Finally, basic technologies are the working elements that actually cooperate in the integrated application. They are the soldiers of an army whose success depends on the quality of the individual training and, not least, on the organization.

The architecture design of an integrated intelligent control system is clearly essential, because of its influence in the development and final performance of the system. If the architecture does not specify some form of behavior, the final system can hardly follow it. Such behavior is specified by determining the subsystems that will compose the final system, as well as the role that they will play and the interfaces that will exist among them. The structures more commonly employed for integrated in-

Integrated Computer-Aided Engineering, 2(3) 163-164 (1995) (c) 1995 John Wiley \& Sons, Inc. telligent control systems are agent-based architectures and blackboard architectures.

Architecture-when it is not simple-means cooperation among subsystems. Each subsystem is usually called a "knowledge source" in blackboard systems and an "agent" in agent-based systems; the cooperation being achieved, respectively, by means of data interchange and service offering-request.

Agent-based architectures are characterized by distributing the control responsibilities, making the subsystems more independent than in the former case: There is no central repository of information, and coupling among subsystems is less tight than among subsystems and blackboard in blackboard based systems. Somehow, the agent interaction resembles the client-server structure used in other areas. In his issue, Fedderwitz and Wittig propose a mechanism for further decoupling of agent activities to enhance real-time properties of expert systems integrated in control systems.

Integrated methodologies provide the software support for interaction among subsystems. Though they usually offer a theoretically general framework, they are frequently related to a specific architecture. An integration methodology can be viewed at several levels of resolution. The two most important ones are the conceptual level and the implementation level. Conceptual level specifications are related to the kind of things that subsystems interchange. Implementation level specifications are related to the actual software implementation of the interchanges.

There are several integration models in this area, but none of them is, at present, established as a standard at the conceptual level: Each integrated application uses the model that is more suitable to its needs. The paper by Alarcon et al. proposes a kind of conceptual specification for real-time, blackboard based applications from the cognitive standpoint. The paper by Leitch et al. shows a new approach to building easily integrable diagnosers: It proposes developing diagnosing applications based on composing software chips.

Concerning the so-called basic technologies, there are many of them that have been used to try out 
their capabilities against plant control problems, both coming from the artificial intelligence and the conventional software engineering communities. Fuzzy systems, neural networks, expert systems, and model-based reasoning have been chosen as clear examples of advanced technologies that are already being used within industrial control applications. In principle, the kind of problems that the first two and the last two deal with are of different nature, covering complementary aspects in intelligent control. In this sense, expert systems and model based reasoning are often associated with specific architectures that try to take as much advantage as possible from their characteristic approach to the problems.

Fuzzy systems offer, among other possibilities, a way to enhance the behavior of somewhat elementary controllers by introducing human knowledge in them. This kind of knowledge embedding is difficult with classic controllers, because they are built with a mathematical template and human brains are not. Control knowledge affected by uncertainty can then be managed and used in real time. You can see examples of this technology even in the daily press. Matía et al., in this issue, use fuzzy logic to build blackboard integrable modules, which perform real-time plant data validation by using heuristic knowledge extracted from operators. This kind of work is of special relevance in control systems.

Neural networks offer a way to get some form of computational knowledge from raw data. By making use of this technology, it is possible to develop advanced systems (for identification, control, prediction, etc.) when there is no explicit knowledge available. This technology also addresses the time-varying character of systems under control, being able to self-tune to new system states. The paper of $\mathrm{Li}$ and Jiang shows the application of neural networks to robot arm cinematic control.

Expert system technology has been the flagship of intelligent control. This is due to the possibilities of expert system shells to model human knowledge, which has made possible quick implementations of computerized expertise-based control. The $\mathrm{R}+\mathrm{D}$ effort in this area has been quite intensive, and as a result we have available several commercial expert system shells that are successfully employed in realtime applications. However, expert systems are quite often related to an architecture, as in Fedderwitz and Wittig, previously mentioned, or to a functional underlying model, as in the Nordvik and Wilikens paper.

Model-based reasoning systems, generally speaking, are an artificial intelligence technology that fo- cuses on the use of deep knowledge about the system under control. This is in contrast with the shallow knowledge approach of other technologies such as expert systems or fuzzy control. Deep knowledge is knowledge about the structure and physical relations existing in the system that is being controlled. The main application of model-based reasoning is in the area of diagnosis, but other applications are being developed, for instance in control or data validation. This kind of deep knowledge can be found in FORMENTOR systems (see the Nordvik and Wilikens paper), where it is used to assess operators in unusual and potentially hazardous situations. Also, in the composing solution of Leitch et al., software modules are identified from a functional analysis of the complete model-based diagnostic process.

And finally, though not included in this issue, we shall mention other technologies, coming from both the conventional and artificial intelligence sides, that are emerging and may be of great interest in this area, such as advanced user interfaces, learning, and hybridization.

To conclude, we would like to say that this issue is aimed at emphasizing the fact that the area of integrated intelligent control is becoming very active due to the general need of using specific technologies to solve specific problems and, subsequently, the need of integrating those techniques. We think that knowledge-based integration is a good and promising starting point, since it can provide a technology-independent way of building integrated applications.

This special issue of Integrated Computer-Aided Engineering contains some papers in the forefront of Integration Technology for Real-Time Intelligent Control Systems. We hope they offer an interesting perspective and prospective view of heterogeneous computer technologies integration for intelligent control systems.

E. de Pablo

INH Repsol, S. A.

Instituto de Ingeniería del Conocimiento, Spain

A. Jimenez

Universidad Politécnica de Madrid, Spain

$V$. López

P. Rodríguez

Instituto de Ingeniería del Conocimiento Dept. de Ingeniería Informática

Universidad Autónoma de Madrid, Spain

Guest Editors 